\title{
TURBULENT FLOW DEVELOPMENT CHARACTERISTICS IN CHANNEL INLETS *
}

\author{
T. Y. NA \\ University of Michigan-Dearborn \\ Dearborn (Mich. 48128), U.S.A. \\ and \\ Y. P. LU \\ Naval Ship Research and Development Center \\ Annapolis (Maryland 21402), U.S.A.
}

\begin{abstract}
The turbulent flow characteristics in the inlet regions of circular pipes and parallel plates are considered separately in this paper. The momentum integral equation of the boundary layer near the wall and the overall continuity equation for the flow are solved. The variation of the shape factor is taken into consideration in the solution by supplementing the two basic equations by an entrainment equation. Numerical solutions and available experimental results show that the present theory is in good agreement with experimental data for flow in both pipe and parallel plates.

\section{Nomenclature}

$a_{11} \ldots a_{34}$ defined in (30)

$A_{1} \ldots A_{14}$ defined in (17)

$b_{11} \ldots b_{23}$ defined in (30)

$H \quad$ the shape factor, $\delta^{*} / \theta$

$L \quad$ half width between parallel plates

$n \quad$ constant in velocity distribution

$r$ radial coordinate

$R \quad$ radius of pipe

Re Reynolds number, $2 R U_{\mathrm{p}_{0}} / \nu$ for pipe flow, and $2 L U_{\mathbf{p}_{0}} / v$ for flow between parallel plates
\end{abstract}

* All opinions or assertions made in this paper are those of the authors and are not to be construed as official or necessarily reflecting the views of the Navy or the naval service at large. 


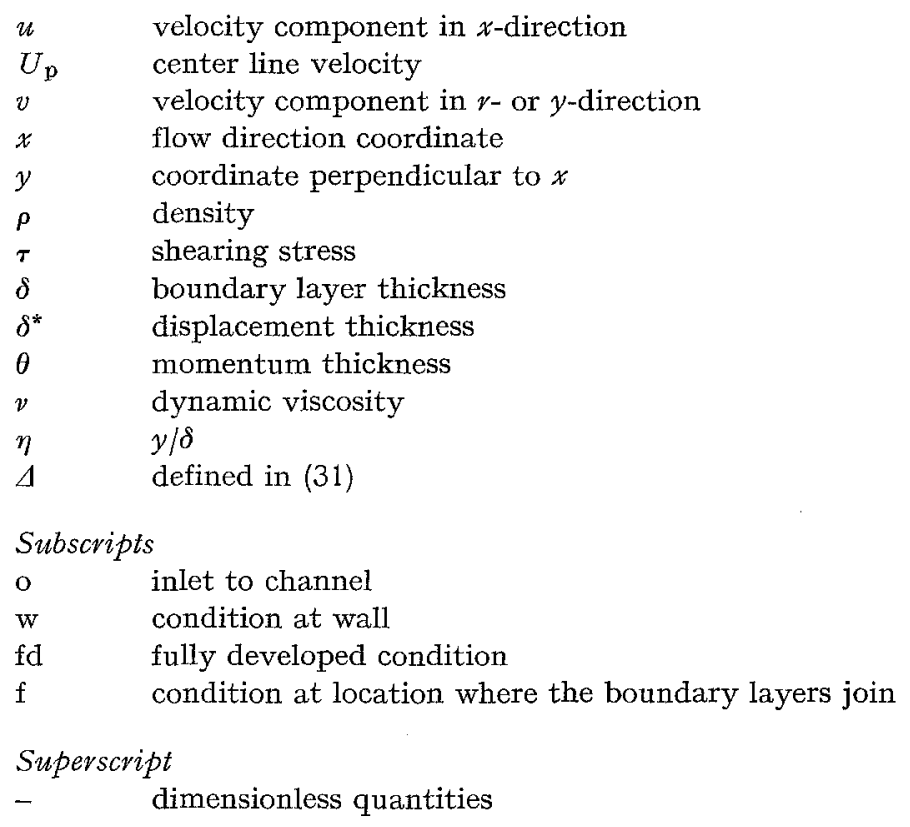

\section{$\S 1$. Introduction}

This paper considers the turbulent flow in the inlet region of circular pipes and parallel plates. The flow at the entrance is assumed to be uniform. Owing to viscous friction, however, a boundary layer will be formed on the wall of the pipe and its width will increase in the downstream direction. The velocity profile consists of a boundary layer profile near the wall joined in the center by a line of constant velocity. Due to the fact that the volume of flow must remain the same for every cross section, the decrease in the rate of flow near the wall must be compensated by a corresponding increase in velocity near the center line. Thus, the boundary layer is formed under the influence of accelerated external flow. At large distance from the entrance, the boundary layer gradually occupies the whole pipe. At the point where the boundary layer reaches the center line, the velocity profile has not reached a fully developed distribution. A short distance further downstream, the flow becomes fully developed.

Analytical prediction of flow characteristics in the inlet region is difficult due to the fact that the region consists of two sections and the treatment by the boundary layer approach can only lead to 
solutions to the first section. No theory has been proposed for the solution of the second section where the boundary layer has merged at the center line, but fully developed turbulent flow has not been established.

In the present paper, the first section of the inlet region is solved by using a more detailed boundary layer treatment. The pressure gradient and variation of the shape factor, neglected by other analyses [1-5], have been considered. Comparisons are made with the experimental data of Barbin and Jones [6] for the flow in circular pipes and Byrne, Hatton, and Marriott [7] for the flow between parallel plates. The close agreement with experimental data in comparison with results from other theories shows that the analysis presented in the present paper better predicts the flow characteristics in the inlet section of channels. The two cases of circular pipe flow and parallel plate flow are treated separately.

\section{$\$ 2$. Inlet region of circular pipes}

The steady, incompressible axisymmetric boundary layer equations are well-known and can be written as (Fig. 1)

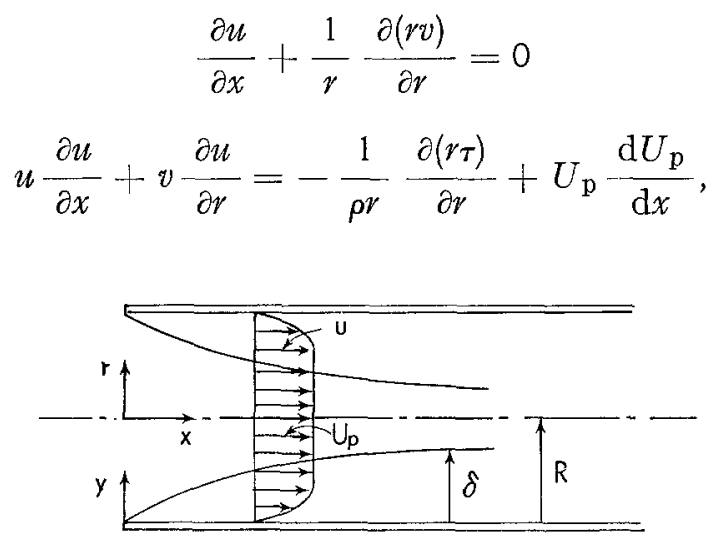

Fig. 1. Schematic diagram of the pipe.

where the velocities are the time-averaged quantities. By nondimensionalizing the variables by

$$
\bar{u}=\frac{u}{U_{\mathrm{p}}(x)}, \quad \bar{U}_{\mathrm{p}}(\bar{x})=\frac{U_{\mathrm{p}}(x)}{U_{\mathrm{p}_{0}}}, \quad \bar{x}=\frac{x}{R}, \quad \bar{r}=\frac{r}{R}
$$


and integrating over the pipe radius, eqs. (1) and (2) become

$$
\begin{gathered}
\frac{\partial}{\partial \bar{x}}\left[\bar{U}_{\mathrm{p}} \int_{0}^{1} \bar{u} \bar{r} \mathrm{~d} \bar{r}\right]=0 \\
\frac{\partial}{\partial \bar{x}} \int_{0}^{1} \bar{u}(1-\bar{u}) \bar{r} \mathrm{~d} \bar{r}+ \\
+\frac{2}{\bar{U}_{\mathrm{p}}} \frac{\mathrm{d} \bar{U}_{\mathrm{p}}}{\mathrm{d} \bar{x}} \int_{0}^{1} \bar{u}(1-\bar{u}) \bar{r} \mathrm{~d} \bar{r}+ \\
+\frac{1}{\bar{U}_{\mathrm{p}}} \frac{\mathrm{d} \bar{U}_{\mathrm{p}}}{\mathrm{d} \bar{x}} \int_{0}^{1}(1-\bar{u}) \bar{r} \mathrm{~d} \bar{r}=\frac{\tau_{\mathrm{w}}}{\rho \bar{U}_{\mathrm{p}}^{2}} .
\end{gathered}
$$

The next step is to select a velocity profile and a proper law of friction. In the work of Bowlus and Brighton [5], a 1/7-power law velocity profile and the Schultz-Grunow wall shear relation for flat plate flow with zero pressure gradient were selected [8]. Such a choice simplifies the mathematics in that two equations will suffice for the solution of the problem, since the shape factor $H\left(=\delta^{*} / \theta\right)$ has a constant value of $9 / 7$, thus eliminating one differential equation for the solution of $H$.

Since the flow in the entrance region is an accelerating flow which does create a nonzero pressure gradient, the use of Schultz-Grunow's friction law is inherently in error due to the fact that it can only be applied to boundary layer flow over flat plates without a pressure gradient. Furthermore, the local Reynolds number in the boundary layer far exceeds the upper limit of the Reynolds number for the 1/7-power law velocity profile to apply. Therefore, both equations have to be replaced by equations without such limitations. In this work, the velocity profile chosen is the general power law profile defined by

$$
\frac{u}{U_{\mathbf{p}}}=\left(\frac{y}{\delta}\right)^{n} \quad \text { or } \quad \bar{u}=\left(\frac{\bar{y}}{\bar{\delta}}\right)^{n},
$$

where $\delta$ is the boundary layer thickness, and the coordinate $y$ is related to the radial axis $r$ by $y=\mathrm{R}-r$. The dimensionless form of these new variables is defined similar to eq. (3) as

$$
\bar{y}=\frac{y}{\mathrm{R}}, \quad \bar{\delta}=\frac{\delta}{\mathrm{R}} .
$$

For the velocity profile given by eq. (6), it can be shown that the displacement and momentum thicknesses are given by 


$$
\frac{\delta^{*}}{\delta}=\frac{n}{n+1} \text { and } \frac{\theta}{\delta}=\frac{n}{(n+1)(2 n+1)} .
$$

Thus, the power $n$ can be related to the shape factor by the following equation

$$
H=\frac{\delta^{*}}{\theta}=2 n+1
$$

For turbulent boundary layer flow with a pressure gradient, the shearing stress at the wall can be approximated quite well by the well-known empirical formula of Ludwieg and Tillman [8]* as

or

$$
\frac{\tau_{\mathrm{W}}}{\rho U_{\mathrm{p}}^{2}}=0.123 \times 10^{-0.678 H}\left(\frac{U_{\mathrm{p}} \theta}{\nu}\right)^{-0.268}
$$

$$
\frac{\tau_{\mathrm{w}}}{\rho U_{\mathbf{p}}^{2}}=0.123 \times 10^{-0.6 .68 H}\left(\bar{U}_{\mathrm{p}} \frac{\operatorname{Re}}{2} \bar{\theta}\right)^{-0.268},
$$

where $\operatorname{Re}$ is defined as $2 \mathrm{R} U_{\mathrm{p}_{0}} / v$, and $\bar{\theta}=\theta / \mathrm{R}$.

The introduction of the shape factor $H$ necessitates another equation for its solution. This equation is usually referred to as the entrainment equation. Various forms of this equation have been proposed (see, e.g., the review article by Rotta [9] for a summary of these equations). It was decided, however, that the equation by von Doenhoff and Tetervin [8] would be used in this work for its proven accuracy in predicting boundary layer characteristics. This equation can be written as

$\vec{\theta} \frac{\mathrm{d} H}{\mathrm{~d} \bar{x}}=\mathrm{e}^{4.680(H-2.975)}\left[-\frac{\bar{\theta}}{\bar{U}_{\mathrm{p}}} \frac{\mathrm{d} \bar{U}_{\mathrm{p}}}{\mathrm{d} \bar{x}}\left(\frac{\rho U_{\mathrm{p}}^{2}}{\tau_{0}}\right)-2.035(H-1.286)\right]$,

where the quantity $\tau_{0} / p U_{\mathrm{p}}^{2}$ in eq. (11), according to Doenhoff and Tetervin [8], is evaluated from the equation by Squire and Young [8]

$$
\frac{\tau_{0}}{\rho U_{\mathbf{p}}^{2}}=\frac{0.0288}{\left[\log \left(4.075 \frac{U_{\mathbf{p}} \theta}{v}\right)\right]^{2}} .
$$

* For fully developed flow, we have Blasius' law [8].

$$
\frac{\tau_{\mathrm{w}}}{\rho u_{\mathrm{qd}}{ }^{2}}=\frac{0.03955}{(\mathrm{Re})^{0.25}} \text {. }
$$

In the calculation, eq. (10a) was used first until $\tau_{\mathrm{w}}$ dropped to the fully developed value given by eq. (10c). From that section on, eq. (10c) was used. 
Combining eqs. (11) and (12), we obtain the final form of the entrainment equation

$$
\begin{gathered}
\bar{\theta} \frac{\mathrm{d} H}{\mathrm{~d} \bar{x}}=\mathrm{e}^{4.680(H-2.975)}\left\{-\frac{\bar{\theta}}{\bar{U}_{\mathrm{p}}} \frac{\mathrm{d} \bar{U}_{\mathrm{p}}}{\mathrm{d} \bar{x}} \frac{\left[\log \left(2.0375 \operatorname{Re} \bar{U}_{\mathrm{p}} \bar{\theta}\right)\right]^{2}}{0.0288}+\right. \\
-2.035(H-1.286)\} .
\end{gathered}
$$

By substituting the velocity profile, eq. (6), into eqs. (4) and (5) and, together with eq. (13), solving for the three differentials $\mathrm{d} \bar{\delta} / \mathrm{d} \bar{x}, \mathrm{~d} \bar{U}_{\mathbf{p}} / \mathrm{d} \bar{x}$, and $\mathrm{d} H / \mathrm{d} \bar{x}$, the following system of differential equations is obtained

$$
\begin{gathered}
-\frac{\mathrm{d} \bar{U}_{\mathrm{p}}}{\mathrm{d} \bar{x}}=\frac{2 A_{2} A_{8} A_{10}+2 A_{2} A_{7} A_{14}+A_{3} A_{14} A_{6}}{2 A_{1} A_{6} A_{10}+2 A_{2}\left(A_{7} A_{13}-A_{9} A_{10}\right)+A_{3} A_{13} A_{6}} \\
\frac{\mathrm{d} \bar{\delta}}{\mathrm{d} \bar{x}}=\frac{1}{A_{6} A_{10}}\left[A_{8} A_{10}+A_{7} A_{14}-\left(A_{7} A_{13}-A_{9} A_{10}\right) A_{15}\right] \\
\frac{\mathrm{d} H}{\mathrm{~d} \bar{x}}=-\frac{A_{14}}{A_{10}}+\frac{A_{13} A_{15}}{A_{10}}
\end{gathered}
$$

where

$$
\begin{aligned}
& A_{1}=\frac{1}{2}(1-\bar{\delta})^{2}+\bar{\delta}\left(\frac{1}{n+1}-\frac{\bar{\delta}}{n+2}\right) \\
& A_{2}=\frac{\bar{U}_{\mathrm{p}}}{n+1}-\bar{U}_{\mathrm{p}}(1-\bar{\delta})-\frac{2 \bar{\delta} \bar{U}_{\mathrm{p}}}{n+2} \\
& A_{3}=\frac{\bar{U}_{\mathrm{p}} \bar{\delta}}{(n+1)^{2}}-\frac{\bar{U}_{\mathrm{p}} \bar{\delta}^{2}}{(n+2)^{2}} \\
& A_{4}=\frac{1}{n+1}-\frac{1}{2 n+1}-\frac{\bar{\delta}}{n+2}+\frac{\bar{\delta}}{2 n+2} \\
& A_{5}=\bar{\delta}\left(\frac{n}{n+1}-\frac{\bar{\delta}}{2}+\frac{\bar{\delta}}{n+2}\right) \\
& A_{6}=A_{4}-\frac{\bar{\delta}}{n+2}+\frac{\bar{\delta}}{2(n+1)} \\
& A_{7}=\frac{\bar{\delta}^{2}}{2(n+2)^{2}}-\frac{1}{2(n+1)^{2}}+\frac{\bar{\delta}}{(2 n+1)^{2}}-\frac{\bar{\delta}^{2}}{4(n+1)^{2}}
\end{aligned}
$$




$$
\begin{aligned}
& A_{8}=\frac{\tau_{0}}{\rho U_{\mathrm{p}}^{2}} \\
& A_{9}=\frac{2 \bar{\delta} A_{4}}{\bar{U}_{\mathrm{p}}}+\frac{A_{5}}{\bar{U}_{\mathrm{p}}} \\
& A_{10}=\left(\bar{U}_{\mathrm{p}} \frac{\operatorname{Re}}{2} \bar{\theta}\right)^{\mathrm{t}} \theta \\
& A_{11}=A_{10} / \bar{U}_{\mathrm{p}} \\
& A_{12}=0.0135(H-1.4) \\
& A_{13}=\mathrm{e}^{5(H-1.4)} A_{11} \\
& A_{14}=\mathrm{e}^{5(H-1.4)} A_{12} .
\end{aligned}
$$

\section{§ 3. Starting condition}

In the experimental work of Barbin and Jones [6], turbulence was promoted by a 1-inch strip of sparsely distributed sand grains glued to the pipe wall 2 inches downstream of its leading edge. At a distance of 2 inches, the local Reynolds number is $9.7 \times 10^{4}$ (for their data with $\operatorname{Re}_{D}=3.88 \times 10^{5}$ ) which is slightly below the generally recognized critical Reynolds number for transition. It is therefore reasonable to assume that the boundary layer is laminar from the leading edge to the distance of 2 inches and from there on, due to the effect of roughness, it becomes turbulent. At the distance of 2 inches (corresponding to $\bar{x}=0.5$ ), the momentum thickness for the laminar boundary layer immediately upstream of transition can be estimated by Blasius' solution as

$$
\theta(\operatorname{lam})=0.664 \sqrt{\frac{2 \bar{x}}{\operatorname{Re}}}=0.00107 .
$$

On the other hand, the momentum, thickness for the turbulent boundary layer immediately downstream of transition can be estimated by the $1 / 7$-power law for flat plate as

$$
\begin{aligned}
\bar{\theta}(\text { tur }) & =0.036 \bar{x}_{\text {tr }}\left(\frac{2}{\operatorname{Re} \bar{x}_{\text {tr }}}\right)^{1 / 5} \\
& =0.00315 \bar{x}_{\mathbf{t r}}^{4 / 5},
\end{aligned}
$$

where $\bar{x}_{\text {tr }}$ is a pseudodistance. 
At transition, the first condition to be satisfied is that the momentum thickness remains constant. Equating $\bar{\theta}$ from eqs. (18) and (19), we get

$$
\bar{x}_{\mathrm{tr}}=0.258 \text {. }
$$

At this value of $\bar{x}_{\text {tr }}$, the boundary layer thickness is estimated to be

$$
\bar{\delta}(\text { tur })=(72 / 7) \bar{\theta}=0.011 .
$$

The shape factor upstream of transition is estimated by Blasius' solution as 2.586. Across transition, the decrease in the shape factor is estimated to be 1.3, based on Fig. 22.13 of Schlichting's book [8]. Thus, the shape factor downstream of transition is 1.286.* Due to the short distance from entrance to transition, $\bar{U}_{\mathrm{p}}$ is taken to be approximately unity. The starting conditions for the integration of eqs. (14), (15), and (16) are then

$$
\begin{array}{cl}
\bar{x}=0.5: & \bar{\delta}=0.011 \\
& H=1.286 \\
& \bar{U}_{\mathrm{p}}=1 .
\end{array}
$$

It should be emphasized that, in the integration process, $\bar{x}_{\mathrm{tr}}=0.258$ is actually used in eq. (20). However, the results are presented in terms of the physical distance $\bar{x}$.

\section{§ 4. Comparison with data of barbin and jones}

Equations (14), (15), and (16), subjected to the initial conditions given by eq. (20), are integrated by Runge-Kutta's method. The results give $\bar{U}_{\mathrm{p}}, \bar{\delta}$, and $H$ as functions of $\bar{x}$. Fig. 2 shows a comparison between the theory and the experimental data of Barbin and Jones [6]. The solid line represents the calculated $\bar{U}_{\mathrm{p}}$ as a function of $\bar{x}$. It is seen to correlate more closely with the data than that of Bowlus and Brighton [5]. The results show that the turbulent boundary layer thickness equals the radius of the pipe at an axial distance of 57 radii, beyond which point the solution based on a boundary layer assumption ceases to apply. If the center line velocity and pressure at this point are denoted by $U_{\mathrm{pp}}$

\footnotetext{
* The value of $H=1.286$ corresponds to a $1 / 7$-power law velocity profile (see, e.g., eq. (21.6) of [8]).
} 


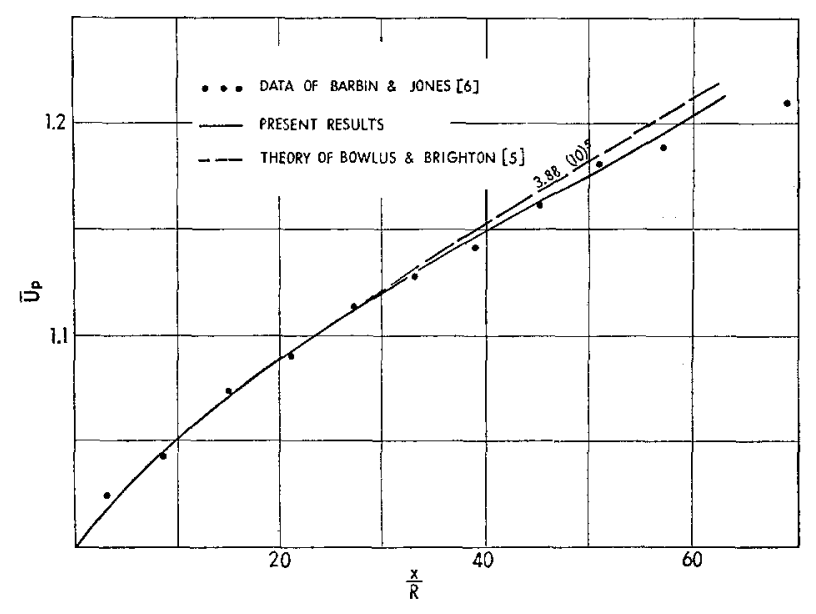

Fig. 2. Comparison of $\bar{U}_{\mathrm{p}}(\bar{x})$ with experimental data.

and $p_{\mathrm{f}}$, respectively, Bernoulli's equation can be used to obtain

$$
\frac{p-p_{\mathrm{p}}}{\rho \bar{u}^{2}}=\frac{U_{\mathrm{pf}}^{2}-U_{\mathrm{p}}^{2}}{2 \bar{u}^{2}}=\frac{1}{2}\left(\bar{U}_{\mathrm{pf}}^{2}-\bar{U}_{\mathrm{p}}^{2}\right) .
$$

Experimental data of Barbin and Jones [6] are again compared with the results calculated by eq. (21) and are shown in Fig. 3. The agreement is extremely close.

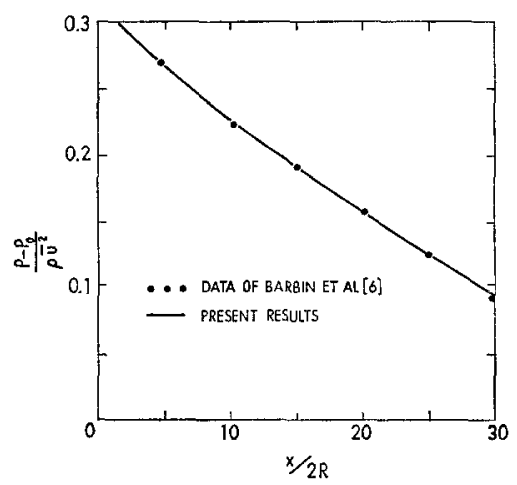

Fig. 3. Comparison of pressure variation with experimental data $\left(\operatorname{Re}=3.88 \times 10^{5}\right)$. 
The ratio of wall skin friction to its fully developed value is given by

$$
\begin{aligned}
\frac{\tau_{\mathrm{W}}}{\tau_{\mathrm{w}_{\mathrm{fd}}}} & =\frac{0.123 \times 10^{-0.678 H}\left(\bar{U}_{\mathrm{p}} \frac{\operatorname{Re}}{2} \bar{\theta}\right)^{-0.268} \rho U_{\mathrm{p}}^{2}}{0.03955\left(\operatorname{Re}^{-0.25}\right) \rho \bar{u}_{\mathrm{fd}}^{2}} \\
& =3.75(10)^{-0.678 \mathrm{H}} \operatorname{Re}^{-0.018} \bar{U}_{\mathrm{p}}^{1.732} \bar{\theta}^{-0.268}
\end{aligned}
$$

for $\tau_{\mathrm{w}} / \tau_{\mathrm{w}_{\mathrm{fa}}} \geq 1$. Fig. 4 shows the ratio calculated from eq. (22) compared with experimental data. Fully developed friction was found to be reached at an axial distance of approximately 17 diameters which is very close to the value obtained by Barbin and Jones.

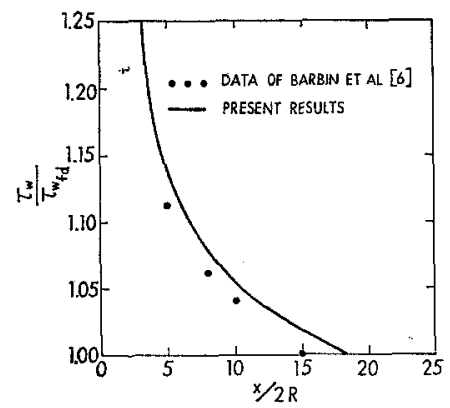

Fig. 4. Comparison of axial friction distribution with experimental data $\left(\operatorname{Re}=3.88 \times 10^{5}\right)$.

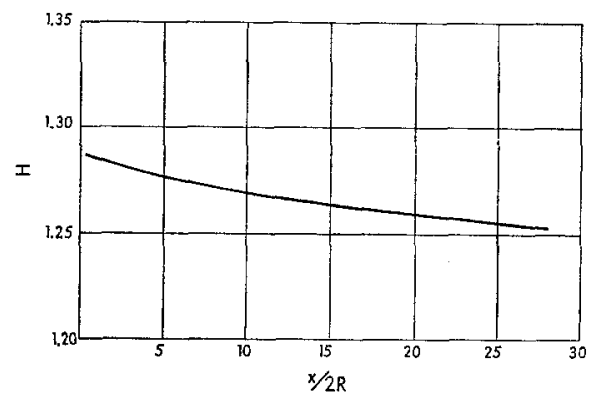

Fig. 5. Variation of $H$ with $\bar{x}\left(\operatorname{Re}=3.88 \times 10^{5}\right)$.

Finally, the shape factor variation in the flow direction is plotted in Fig. 5. This shows that the shape factor $H$ changes from 1.286 to 1.25. Since these values of $H$ correspond to velocity distributions 
varying continuously from 1/7-power to 1/8-power laws, the assumptions of 1/7-power velocity distribution and constant shape factor at 1.286 are bound to lead to error.

\section{§ 5. Inlet region of parallel plates}

Following exactly the same steps as in the analysis of the inlet region of a circular pipe, the theory is applied to flow between parallel plates. In terms of the dimensionless quantities

$$
\bar{u}=\frac{u}{U_{\mathrm{p}}}, \quad \bar{U}_{\mathrm{p}}=\frac{u}{U_{\mathrm{p}_{0}}}, \quad \bar{x}=\frac{x}{L}, \quad \bar{y}=\frac{y}{L}
$$

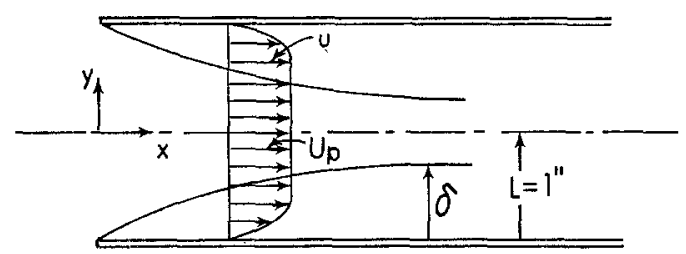

Fig. 6. Schematic diagram of parallel plates.

and Fig. 6, the momentum, continuity, and entrainment equations, can be written as

$$
\begin{aligned}
& \frac{\partial}{\partial \bar{x}} \int_{0}^{1} \bar{u}(1-\bar{u}) \mathrm{d} \bar{y}+\frac{2}{\bar{U}_{\mathrm{p}}} \frac{\mathrm{d} \bar{U}_{\mathrm{p}}}{\mathrm{d} \bar{x}} \int_{0}^{1} \bar{u}(1-\bar{u}) \mathrm{d} \bar{y}+ \\
& +\frac{1}{\bar{U}_{\mathrm{p}}} \frac{\mathrm{d} \bar{U}_{\mathrm{p}}}{\mathrm{d} \bar{x}} \int_{0}^{1}(1-\bar{u}) \mathrm{d} \bar{y}=\frac{\tau_{\mathrm{w}}}{\rho U_{\mathrm{p}}^{2}} \\
& \frac{\partial}{\partial \bar{x}}\left\{\bar{U}_{\mathbf{p}} \int_{0}^{1} \bar{u} \mathrm{~d} \bar{y}\right\}=0 \\
& \frac{\mathrm{d} H}{\mathrm{~d} \bar{x}}=\mathrm{e}^{4.680(H-2.975)} \times \\
& \times\left\{-\frac{1}{\bar{U}_{\mathrm{p}}} \frac{\mathrm{d} \bar{U}_{\mathrm{p}}}{\mathrm{d} \bar{x}} \frac{\left[\log _{10}\left(2.0375 \operatorname{Re} \bar{U}_{\mathrm{p}} \frac{(H-1) \bar{\delta}}{H(H+1)}\right)\right]^{2}}{0.0288}+\right. \\
& \left.-2.035(H-1.286) \frac{H(H+1)}{\bar{\delta}(H-1)}\right\}
\end{aligned}
$$


where $\tau_{\mathrm{w}}$ is given by Ludwieg and Tillman's relation

$$
\begin{aligned}
\frac{\tau_{\mathrm{w}}}{\rho U_{\mathrm{p}}^{2}}=0.123 \times 10^{-0.678 H}\left(\bar{U}_{\mathrm{p}} \frac{\operatorname{Re}}{2} \bar{\theta}\right)^{-0.268} \\
=0.123 \times 10^{-0.678 H}\left(\bar{U}_{\mathrm{p}} \frac{\operatorname{Re}}{2} \frac{(H-1) \bar{\delta}}{H(H+1)}\right)^{-0.268} .
\end{aligned}
$$

By using the general velocity distribution given by

$$
\bar{u}=\eta^{n},
$$

where $n$ is related to the shape factor $H$ through the relation $H=$ $=2 n+1$, eqs. (24), (25), and (26) can be solved to obtain the following system of differential equations

$$
\begin{aligned}
\frac{\mathrm{d} \bar{U}_{\mathrm{p}}}{\mathrm{d} \bar{x}} & =\frac{1}{\Delta}\left(b_{14} a_{22}-a_{12} b_{23}\right) \\
\frac{\mathrm{d} \bar{\delta}}{\mathrm{d} \bar{x}} & =\frac{1}{\Delta}\left(b_{11} b_{23}-b_{21} b_{14}\right) \\
\frac{\mathrm{d} H}{\mathrm{~d} \bar{x}} & =a_{34}-a_{33} \frac{\mathrm{d} \bar{U}_{\mathrm{p}}}{\mathrm{d} \bar{x}},
\end{aligned}
$$

where

$$
\begin{aligned}
& a_{11}=\frac{\bar{\delta}}{H(H+1)}-\frac{(H-1) \bar{\delta}}{H^{2}(H+1)^{2}}(2 H+1) \\
& a_{12}=\frac{H-1}{H(H+1)} \\
& a_{13}=\frac{1}{\bar{U}_{\mathrm{p}}}\left[\frac{2(H-1) \bar{\delta}}{H(H+1)}+\frac{(H-1) \bar{\delta}}{H+1}\right] \\
& a_{14}=0.123 \times 10^{-0.678 H}\left[\bar{U}_{\mathrm{p}} \frac{\operatorname{Re}}{2} \frac{(H-1) \bar{\delta}}{H(H+1)}\right]^{-0.268} \\
& a_{21}=-\bar{U}_{\mathrm{p}} \frac{2 \bar{\delta}}{(H+1)^{2}} \\
& a_{22}=\bar{U}_{\mathrm{p}} \frac{1-H}{H+1} \\
& a_{23}=1-\frac{(H-1) \bar{\delta}}{H+1}
\end{aligned}
$$




$$
\begin{aligned}
& a_{31}=1 \\
& a_{33}=\mathrm{e}^{4.680(H-2.975)} \frac{1}{\bar{U}_{\mathrm{p}}} \frac{\left[\log _{10}\left(2.0375 \operatorname{Re} \bar{U}_{\mathrm{p}} \frac{(H-1) \bar{\delta}}{H(H+1)}\right)\right]^{2}}{0.0288} \\
& a_{34}=-\mathrm{e}^{4.680(H-2.975)} 2.035(H-1.286) \frac{H(H+1)}{(H-1) \bar{\delta}} \\
& b_{11}=-a_{11} a_{33}+a_{13} \\
& b_{14}=a_{14}-a_{11} a_{34} \\
& b_{21}=-a_{21} a_{33}+a_{23} \\
& b_{23}=-a_{21} a_{34}
\end{aligned}
$$

and

$$
\Delta=\left|\begin{array}{ll}
b_{11} & a_{12} \\
b_{21} & a_{22}
\end{array}\right| .
$$

The starting conditions were estimated by following the same reasoning as used in the case of circular pipes. The experimental data [7] used in checking the present theory have a transition at $\bar{x}=1$, and the Reynolds number $\operatorname{Re}=2 U_{\mathrm{p}_{0}} L / v$ is 110,500 . The

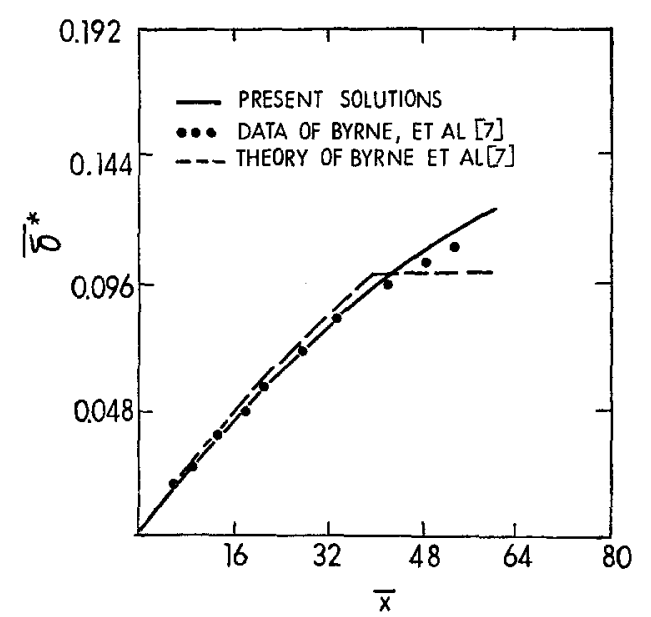

Fig. 7. Comparison of displacement thickness variation with experimental data $(\operatorname{Re}=110,500)$. 


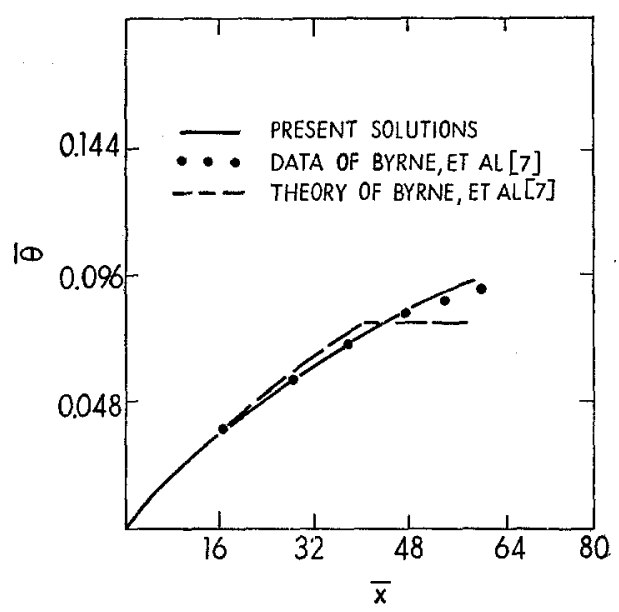

Fig. 8. Comparison of momentum thickness variation with experimental data $(\operatorname{Re}=110,500)$.

starting conditions are found to be*

$$
\bar{x}=1: \quad H=1.286, \quad \bar{\delta}=0.0291, \quad \bar{U}_{\mathbf{p}}=1 .
$$

Numerical solutions are obtained by forward integration, similar to that used in the case of circular pipes. The same degree of accuracy was obtained, as shown in Figs. 7 and 8 for typical results. Solutions by the present method are in better agreement with experimental data than those of Byrne, Hatton, and Marriott [7]. The boundary layers from the walls meet at an axial distance of approximately $\bar{x}=55$, beyond which the boundary layer model ceases to apply.

Received 1 March 1972

\section{REFERENCES}

[1] LATzko, H., Heat Transfer in a Turbulent Liquid or Gas Stream, NACA TM 1068, 1944.

[2] Holphusen, J. S., The Turbulent Boundary Layer in the Inlet Region of Smooth Pipes, Ph.D. thesis, University of Minnesota, 1952.

[3] Deissler, R, G., Trans. ASME 77 (1955) 1221.

* $\bar{x}=1$ corresponds to $\bar{x}_{\mathrm{tr}}=0.638$ and integration proceeds from $\bar{x}_{\mathrm{tr}}=0.638$. The final results are, however, presented in terms of the physical distance $\bar{x}$, as in the case of circular pipes. 
[4] Filippov, G. V., Soviet Physics - Tech. Phys. 32 (1958) 1681.

[5] Bowlus, D. A. and J. A. Brighton, J. Basic Eng. Trans. ASME 90, Ser. D (1968) 431.

[6] Barbin, A. R. and J. B. Jones, J. Nasic Eng. Trans. ASME (1963) 29.

[7] Byrne, J., A. P. Hation and P. G. Marriott, Proc. Inst. of Mech. Engrs. 184 (1969-1970) 697.

[8] Schlichting, H., Boundary Layer Theory, McGraw-Hill Book Company, Inc., 1968, chaps. 20 and 22.

[9] RottA, J. C., Progress in Aeronautical Sciences 2 (1962) 1-220. 